\title{
Power Generation Expansion Planning Using an Interior Point with Cutting Plane (IP/CP) Method
}

\author{
Moon, Guk-Hyun ${ }^{1}$, Seo, In-Yong ${ }^{1}$ and Jaehee, Lee $^{2}$ \\ 1. Power System Research Team, Economy \& Management Research Institute of KEPCO, Naju-si, Jeollanam-do 58217, Korea \\ 2. Department of Electrical and Electronics Engineering, Gwangju University, Gwangju 61743, Korea
}

\begin{abstract}
The generation expansion planning is one of complex mixed-integer optimization problems, which involves a large number of continuous or discrete decision variables and constraints. In this paper, an interior point with cutting plane (IP/CP) method is proposed to solve the mixed-integer optimization problem of the electrical power generation expansion planning. The IP/CP method could improve the overall efficiency of the solution and reduce the computational time. Proposed method is combined with the Bender's decomposition technique in order to decompose the generation expansion problem into a master investment problem and a slave operational problem. The numerical example is presented to compare with the effectiveness of the proposed algorithm.
\end{abstract}

Key words: Benders' decomposition technique, power generation expansion planning, interior point with cutting plane method, mixed integer programming.

\section{Introduction}

The main objectives of the power generation expansion planning are to maintain the reliability of the power system and increase the cost efficient of the electricity energy supply over a given period of time. The power generation expansion planning problem is a complex mixed-integer nonlinear optimization problem which involves a large number of continuous or discrete decision variables and constraints.

The evaluations of the optimum investment capability and the mix of a power generation system require complicated optimization techniques [1]. There have been various attempts to solve the generation expansion problem using dynamic programming [2], Lagrangian relaxation [3], and the MIP (mixed-integer programming) [4-6].

In this study, the power generation expansion planning problem is formulated using an MIP model. The proposed generation expansion problem involves both discrete and continuous variables, which cannot

Correponding author: Guk-Hyun Moon, senior researcher, Ph.D., research field: optimization and power system planning. be incorporated in the same optimization problem [5]. Thus, the Benders' decomposition technique is employed to decompose the original generation planning problem into a discrete expansion investment problem and a non-linear operation problem. Details of the Benders' decomposed optimization technique have been described in Ref. [6].

Because of the complexity of decomposed problems of the generation expansion planning, the estimation of dual variables requires high computational efforts. In this paper, the interior point with cutting plane (IP/CP) method [7, 8] is applied to enhance the computational efficiency of the proposed planning problem. The dual variables of the decomposed problems are updated by the IP/CP method. This IP/CP method is the one of the extended versions of IP (interior point), which improves the performance of the algorithm and solution quality. The computational performance of the IP/CP has been demonstrated in Ref. [8].

The remainder of the paper is organized as follows: Section 2 describes the optimization model for generation expansion planning and required assumptions; Section 3 presents the proposed 
generation expansion planning method with IP/CP; Section 4 presents the numerical results; and Section 5 concludes the paper.

\section{Problem Formulation}

Optimal generation expansion planning aims to minimize investment and operations costs. In this problem, the scales, locations, and periods of investment decisions can be formulated by introducing the MIP technique [4]. The generation investment decisions are scheduled at intervals of one year whereas the electricity demand is met in intervals of one day. Consequently, the generation expansion planning optimization problem can be formulated as follows (1)-(7).

(a) Objective function:

$$
\min _{w g, p} \sum_{y=1}^{Y}\left(\left(\sum_{i \in G+} K G_{i} w g_{i t}+\sum_{d=1}^{D} \sum_{i \in G} c_{G i}^{\omega} p_{\text {Gitd }}\right) \cdot(1+r)^{-(y-1)}\right)
$$

where, $i$ is the index for generation unit, $y$ and $d$ are the index for year and day, respectively; $Y$ and $D$ are the number of planning years and days, $G$ and $G+$ are the set of existing and candidate generation units, respectively; $c_{i}$ is the generation cost coefficient of generation unit $(i)$; $w g$ is the generation investment decisions, $p$ is the dispatch schedules of generation units, $r$ is the discount rate.

(b) Generation investment constraint:

$$
0 \leq w g_{i y}-w g_{i y-1}, \forall i, \forall y
$$

(c) Power balance equation at each node:

$$
\sum_{i \in G} p_{i y d}-\sum_{m, n, k \in \Phi_{m}} f_{m n k y d}=L_{m y d}, \forall m, \forall y, \forall d
$$

where, $f_{\text {mnkyd }}, L_{m y d}, \Phi_{m}$, and $\psi_{m}$ are respectively power flow in transmission line ( $m, n, k)$, system load, set of generation units and transmission lines connected at node $(m)$.

(d) DC power flow equation:

$$
B_{m n k}\left(\delta_{m y d}-\delta_{n y d}\right)=f_{m n k y d}, \forall m n k, \forall y, \forall d
$$

where $B_{m n k}$ and $\delta_{m y h}$ are susceptance of transmission line $(m, n, k)$, scaling factor for the equality, and voltage angle at node $(m)$, respectively. (e) Generation limits of thermal units:

$$
P_{i}^{\min } \cdot w g_{i y} \leq p_{i y d} \leq P_{i}^{\max } \cdot w g_{i y}, \forall i, \forall y, \forall d
$$

where $P^{\min }$ and $P^{\max }$ are minimum and maximum power output of generation unit $(i)$.

(f) Thermal limits of transmission lines:

$$
f_{m n k}^{\max } \leq f_{m n k y d} \leq f_{m n k}^{\max }, \forall m n k, \forall y, \forall d
$$

(g) Power flow variables equality equation:

$$
f_{\text {mnkyd }}=-f_{\text {nmkyd }}, \forall m n k, \forall y, \forall d
$$

As the generation planning problem with MIP technique is formulated, the proposed Benders' decomposition solution technique with the IP/CP method is presented in the following section.

\section{Proposed Planning Method for Generation System with IP/CP Method}

3.1 Benders' Decomposed Generation Expansion Planning Problem

In this sub-section, the power generation expansion planning problem is solved using the Benders' decomposition technique. The investment decision variables involved in the proposed expansion planning problem consists of mixed integer variables that cannot be incorporated within the traditional optimization problem $[5,6]$. In this study, therefore, the Benders' decomposition technique is adopted to separate the combinatorial expansion problem into a master investment problem and a slave operational problem. The master problem for minimizing generation investment costs can be formulated as following Eqs. (8)-(10):

[Master Problem] Generation Expansion Planning:

MP-a) Objective function for minimizing the generation investment and operational dual cost:

$$
\min _{w g} \sum_{y=1}^{Y} \sum_{i \in G+}\left(K G_{i} w g_{i t}+\phi_{y}\right) \cdot(1+r)^{-(y-1)}
$$

MP-b) Dual constraint for investment variables:

$$
\phi_{y} \geq \Theta_{y}^{(n)}+\Phi_{i y}^{(n)} \cdot w g_{i y}, \forall y
$$

where, $\Theta$ and $\Phi$ are the respectively the marginal 
value of the objective function, the generation capacity limit in the slave problem; and $n$ is the index for the iteration number.

MP-c) Generation investment constraint:

$$
0 \leq w g_{i y}-w g_{i y-1}, \forall i, \forall y
$$

Given an optimized generation investment plan (wg*), the slave problem of the fault current level calculation can be derived as the following optimization problem (11)-(15):

[Slave Problem] Power System Operation:

$\mathrm{SP}-\mathrm{a})$ Objective function for minimizing operation cost:

$$
\min _{p} \sum_{y=1}^{Y} \sum_{d=1}^{D} \sum_{i \in G} c_{G i}^{\omega} p_{\text {Gitd }} \cdot(1+d)^{-(y-1)}
$$

SP-b) Power balance equation at each node:

$$
\sum_{i \in G} p_{i y d}-\sum_{m, n, k \in \Phi_{m}} f_{m n k y d}=L_{m y d}, \forall m, \forall y, \forall d
$$

SP-c) DC power flow equation:

$$
B_{m u k}\left(\delta_{m y d}-\delta_{\text {nyd }}\right)=f_{m u k y d}, \forall m n k, \forall y, \forall d
$$

SP-d) Generation limits of thermal units:

$$
P_{i}^{\min } \cdot w g_{i y} \leq p_{i y d} \leq P_{i}^{\max } \cdot w g_{i y}, \forall i, \forall y, \forall d
$$

SP-e) Thermal limits of transmission lines:

$$
f_{m n k}^{\max } \leq f_{m n k y d} \leq f_{m n k}^{\max }, \forall m n k, \forall y, \forall d
$$

SP-f) Power flow variables equality equation:

$$
f_{\text {mnkyd }}=-f_{\text {nmkyd }}, \forall m n k, \forall y, \forall d
$$

The dual constraint (9) is represented in terms of the expected marginal value of the system operational constraints (14). The marginal value indicates the optimality and feasibility of the sub-problem. As the Benders' decomposed generation expansion problem which incorporating dual variables are formulated, the dual optimization technique with the IP/CP method is presented in the following sub-section.

\subsection{Dual Optimization with Interior Point with Cutting Plane (IP/CP) Method}

While the iterations, dual variables are updated adequately based on former iterations step's information. Updating dual multipliers during the iterative process is also critical for the convergence of the Benders' decomposed optimization problem. In this paper, IP/CP Lagrangian updating method [9] is adopted to compute the step length of the dual multiplier at the $i$-th iteration. The IP/CP method could reduce the computational efforts involved in the estimation of global optimization solutions. With the IP/CP method, the marginal value of the generation capacity constraints is used to calculate the step length for the multiplier. The step length of the dual multiplier can be estimated using the following equation:

$$
\max \left\{\sum_{v} \log \alpha_{v} \mid \alpha=b-A x, \quad s>0\right\}
$$

where,

$$
A=\left[\begin{array}{cc}
1 & -l_{v} \\
-1 & 0 \\
0 & -I \\
0 & I
\end{array}\right], \quad x=\left[\begin{array}{l}
z \\
\alpha
\end{array}\right], \quad c=\left[\begin{array}{c}
f\left(\alpha^{n}\right) \\
0 \\
0 \\
\alpha_{\max }
\end{array}\right] .
$$

The element $I$ is an identity vector with the appropriate length, $l_{v}$ is the gradient of dual function with respect to $\lambda_{v}$. Once the value of $\alpha_{v}$ is updated, the slave problem $v$ sends it to the master problem and finds new values for dual variables, and $n$ represents the number of iterations. The tie-line power flow mismatch information is used to calculate the step length for the multiplier. The following equation is used to update the dual variables:

$$
\begin{gathered}
\Theta^{n+1}=\Theta^{n}+\alpha_{\Theta}^{n} \\
\Phi^{n+1}=\Phi^{n}+\alpha_{\Phi}^{n}
\end{gathered}
$$

The step length $\alpha^{n}$ obtained by the IP/CP method is used to update the dual variables. The partitioning of the feasible region by the cutting plane method results in efficient and accurate estimation in dual planes. An in-depth discussion of IP/CP techniques and the issues involved in designing optimization models has been provided in Ref. [8]. The iterative procedure to estimate the optimal solution of the dual 
function with IP/CP is illustrated in Fig. 1.

\subsection{Proposed Generation Capacity Planning Method Incorporating $I P / C P$}

The proposed algorithm procedure is presented as follow, which is used for solve the Benders' decomposed generation expansion problem by the IP/CP method. The algorithms include an iterative process with following steps.

Step 1 -Set the iteration counter $n=1$. Set the primal decision variables to 0 and set the cut variables $\phi, \Phi, \Theta$ to some initial points;

Step 2-Solve the master generation expansion problem subject to planning constraints and determine investment decision variables;

Step 3-Calculate the lower bound of the optimization problem by using the following equation:

$$
L B^{n}:=\sum_{y=1}^{Y} \sum_{i \in G^{+}}\left(K G_{i} w g_{i t}+\phi_{y}\right) \cdot(1+r)^{-(y-1)}
$$

Step 4-Solve the slave power operation problem subject to operation constraints and determine dual variables;

Step 5-Calculate the upper bound of the optimization problem by using the following equation:

$$
U B^{n}:=\sum_{y=1}^{Y}\left(\left(\sum_{i \in G+} K G_{i} w g_{i t}+\sum_{d=1}^{D} \sum_{i \in G} c_{\text {Gi }}^{\omega} p_{\text {Gitd }}\right) \cdot(1+r)^{-(y-1)}\right)(21)
$$

Step 6-Calculate the step length for each dual variable by using Eq. (17);

Step 7-Update the dual constraint parameters in the master problem with step length for each dual variable of the slave problem by using Eqs. (18) and (19);

Step 8-(Stopping criterion) If the obtained duality gap is less than criterion value $\sigma$,

$$
\frac{U B^{n}-L B^{n}}{L B^{n}} \leq \sigma
$$

then the algorithm terminates with investment decision variables $w g^{*}$ as solutions. Otherwise, go back to Step 2 and repeat the algorithm. The overall

\begin{tabular}{|c|c|c|}
\hline $\begin{array}{l}\text { Solve the master } \\
\text { problem } \\
\text { Investment \& dual } \\
\text { cost minimization }\end{array}$ & $\left\{\begin{array}{l}Z_{m p}=\min _{w g} \sum_{y=1}^{Y} \sum_{i \in G+}\left(K G_{i},\right. \\
\text { subject to: }\left\{\begin{array}{l}\text { Investment } \\
\frac{\text { Set of dual }}{\text { (=Maximun }}\end{array}\right.\end{array}\right.$ & $\begin{array}{l}\left.{ }_{i t}+\phi_{y}\right) \cdot(1+r)^{-(y-1)} \\
\text { nstraints } \\
\text { nstraints } \\
\text { apacity limit) }\end{array}$ \\
\hline $\begin{array}{c}\text { Determine } \\
\text { investment } \\
\text { decision variable }\end{array}$ & $\begin{array}{l}\text { Generation unit } \\
\text { investment capacity }\end{array}$ & $\begin{array}{l}\text { Generation unit } \\
\text { investment schedule }\end{array}$ \\
\hline $\begin{array}{l}\text { Solve the slave } \\
\text { problem } \\
\text { System operation }\end{array}$ & \multicolumn{2}{|c|}{ 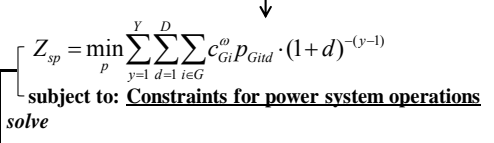 } \\
\hline \multirow{4}{*}{$\begin{array}{c}\text { Dual } \\
\text { optimization } \\
\text { Generation of new } \\
\text { Benders cut }\end{array}$} & \multicolumn{2}{|c|}{ Obtain dual value of slave problem } \\
\hline & $\begin{array}{l}\text { Determine the margin } \\
\text { of generation capacity }\end{array}$ & $\begin{array}{c}\text { Extract dual value of } \\
\text { objective problem }\end{array}$ \\
\hline & & \\
\hline & $\begin{array}{l}\text { Calculate step length } \\
\text { for each dual variables }\end{array}$ & $\begin{array}{l}\text { Add the Benders cut } \\
\text { to the master problem }\end{array}$ \\
\hline
\end{tabular}
procedure of proposed planning method is illustrated in Fig. 1.
Fig. 1 Overall procedure of the proposed planning method.

\section{Numerical Examples}

The proposed generation expansion planning method is tested on modified Garver's 6-bus test system, which is shown in Fig. 2. The length of planning horizon to be considered is set to 5 years, and the average annual increase rate of electricity peak demand during planning horizon is assumed to be $3.5 \%$. The discount rate for calculating present value is set to $5.0 \%$.

It can be seen from Table 1 that the data for the system demand is shown, and Table 2 shows the data for the existing generation unit. Table 3 shows the data for the candidate generation unit.

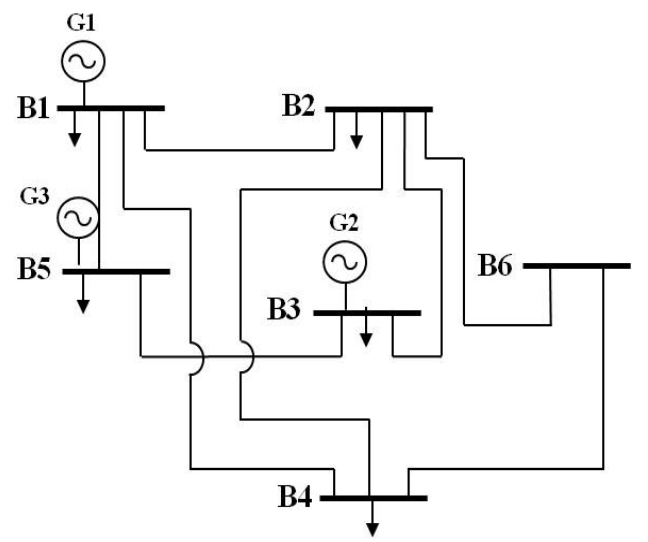

Fig. 2 Modified Garver's 6-bus system. 
Table 1 Data for system demand.

\begin{tabular}{llll}
\hline Bus \# & Demand & Bus \# & Demand \\
\hline B1 & $50 \mathrm{MW}$ & B4 & 85 \\
B2 & $120 \mathrm{MW}$ & B5 & 125 \\
B3 & $20 \mathrm{MW}$ & B6 & 0 \\
& Total Demand & & 400 \\
\hline
\end{tabular}

Table 2 Data for existing generation unit.

\begin{tabular}{llll}
\hline $\begin{array}{l}\text { Generator } \\
\text { number }\end{array}$ & Installed sites & Capacity & $\begin{array}{l}\text { Generation } \\
\text { cost }(\$ / \mathrm{MWh})\end{array}$ \\
\hline G1 & B1 & $150 \mathrm{MW}$ & 40 \\
G2 & B3 & $300 \mathrm{MW}$ & 50 \\
G3 & B5 & $550 \mathrm{MW}$ & 60 \\
\hline
\end{tabular}

Table 3 Data for candidate generation unit.

\begin{tabular}{lllll}
\hline $\begin{array}{l}\text { Generator } \\
\text { number }\end{array}$ & $\begin{array}{l}\text { Candidate } \\
\text { site }\end{array}$ & Capacity & $\begin{array}{l}\text { Generation } \\
\text { cost }(\$ / \mathrm{MWh})\end{array}$ & $\begin{array}{l}\text { Investing } \\
\operatorname{cost}\left(10^{3} \$\right)\end{array}$ \\
\hline G4 & B2 & $280 \mathrm{MW}$ & 52 & 29,000 \\
G5 & B4 & $230 \mathrm{MW}$ & 55 & 22,000 \\
G6 & B6 & $180 \mathrm{MW}$ & 45 & 25,000 \\
\hline
\end{tabular}

The optimal investment decisions are determined by the iterative procedure. The boundary gap tolerance which determines the stopping criterion is set to $10^{-1}$. In addition, the classical gradient based dual optimization method [9] is implemented for the comparison of the computational performance. The investment decision results for each approach are shown in Table 4. Also, Table 5 shows the computation times for the proposed stochastic planning method with IP/CP and the existing optimization method with gradient.

Each approach obtains the feasible solutions which do not violate the power balance constraints and generator lower and upper limit at every planning stage. In addition, the investment decisions by each

Table 4 Cumulative solutions of investment decision.

\begin{tabular}{llllll}
\hline Year & Y1 & Y2 & Y3 & Y4 & Y5 \\
\hline G4 & 0 & 0 & 0 & 0 & 1 \\
G5 & 0 & 0 & 1 & 1 & 1 \\
G6 & 0 & 1 & 1 & 1 & 2 \\
\hline
\end{tabular}

Table 5 Comparison of computation time.

\begin{tabular}{lll}
\hline Approach & $\begin{array}{l}\text { Existing method } \\
\text { with gradient }\end{array}$ & $\begin{array}{l}\text { Proposed method } \\
\text { with IP/CP }\end{array}$ \\
\hline Iterations & 11 & 4 \\
Elapsed times $(\mathrm{ms})$ & 54,034 & 15,938 \\
\hline
\end{tabular}

approach are same. From Table 5, the computational time of the proposed method is $72.17 \%$ decreased compared to the optimization method with gradient. With respect to the iteration number, the iterations number of the IP/CP method is 4 while the iterations number of the gradient method is 17 . These simulation results show that the proposed generation expansion planning method with IP/CP could reduce the computational efforts of the planning problem.

\section{Conclusion}

This paper presents a Benders' decomposition technique to solve the generation expansion planning optimization problem. The IP/CP method was employed to reduce the computational efforts required by the proposed method while maintaining the optimality of the solution. It is demonstrated in numerical examples that the proposed decomposed optimization approach with the IP/CP method effectively reduces the operating time compared to the existing gradient method. In the future, it would be necessary to investigate the combined effects of the other optimization techniques into the generation expansion planning problem.

\section{Acknowledgment}

I would like acknowledge my staff and personnel of the Economy \& Management Research Institute of KEPCO supporting me through research.

\section{References}

[1] Zhu, J., and Chow, M. Y. 1997. "A Review of Emerging Techniques on Generation Expansion Planning." IEEE Trans. on Power Systems 12 (4): 1722-8.

[2] Park, Y. M., Park, J. B., and Won, J. R. 1998. "A Hybrid Genetic Algorithm/Dynamic Programming Approach to Optimal Long-Term Generation Expansion Planning." International Journal of Electrical Power \& Energy Systems 20 (4): 295-303.

[3] Chen, H., Wang, X., and Zhao, X. 2004. "Generation Planning Using Lagrangian Relaxation and Probabilistic Production Simulation." International Journal of Electrical Power \& Energy Systems 26 (8): 597-605.

[4] Dehghan, S., Amjady, N., and Kazemi, A. 2014. 
"Two-Stage Robust Generation Expansion Planning: A Mixed Integer Linear Programming Model." IEEE Trans. on Power Systems 29 (2): 584-97.

[5] Kim, H., Sohn, H. S., and Bricker, D. L. 2011. "Generation Expansion Planning Using Benders' Decomposition and Generalized Network." International Journal of Industrial Engineering 18 (1): 25-39.

[6] Sirikum, J., Techanitisawad, A., and Kachitvichyanukul, V. 2007. "A New Efficient GA-Benders' Decomposition Method: For Power Generation Expansion Planning With Emission Controls.” IEEE Trans. on Power Systems 22
(3): 1092-100.

[7] Aguado, J. A., and Quintana, V. H. 2001. "Inter-Utilites Power-Exchange Coordination: A Market-Oriented Approach.” IEEE Trans. on Power Systems 16 (3): 513-9.

[8] Mitchell, J. E. 2000. "Computational Experience with an Interior Point Cutting Plane Algorithm." SIAM Journal on Optimization 10 (4): 1212-27.

[9] Moon, G. H., Joo, S. K., Hur, D., Jeong, H. S., Ryu, H. S., and Cho, K. W. 2009. "Stochastic Integrated Generation and Transmission Planning Method with Gradient Radar Step (GRS).” Presented at the IEEE T\&D, Seoul, Korea. 